\title{
GNSS Positioning in Non-line-of-Sight Context-a Survey for Technological Innovation
}

\author{
Julia Breßlet ${ }^{*} 1$, Marcus Obst ${ }^{2}$ \\ ${ }^{1}$ Chair for Innovation Research and Technology Management, Technische Universität, 09107 Chemnitz, Germany \\ ${ }^{2}$ BASELABS GmbH, 09126, Chemnitz, Germany
}

\begin{tabular}{l} 
A R T I C L E I N F O \\
\hline Article history: \\
Received: 10 April, 2017 \\
Accepted: 12 May, 2017 \\
Online: 16 June, 2017 \\
\hline Keywords: \\
Non-Light-of-Sight \\
GNSS / Glonass Positioning \\
Urban Areas
\end{tabular}

\begin{abstract}
A B S T R A C T
Reliable, available and accurate localization of people and vehicles is one crucial requirement for intelligent transportation systems (ITSs). This has led to a variety of technical approaches in recent years. Towards an exact positioning via Global Navigation Satellite Systems (GNSSs) the visibility of satellites is an important factor. In this paper, we review the literature regarding the positioning under non-line-of-sight conditions and analyze a range of topics from explicit modeling to empirical data collection. The variety of research indicates a growing interest in robust localization in urban areas. Furthermore, we develop a taxonomy of the technical design for GNSS applications dealing with non-line-of-sight and a typology of the measuring instruments. Both is based on an extensive analysis and review of the state-of-the-art. In doing so, we characterize the current research road map. Thus, the goal of this work is to provide a starting point in terms of the state-of-the-art for further research activities in robust GNSS positioning and the opportunity for technological innovations to ITS.
\end{abstract}

\section{Introduction}

This paper is an extension of work originally presented in the international conference on Intelligent Transportation Systems 2016 [1].

Localization and navigation are two key features for our industrial and globally connected world. Reliable knowing the ego position at any time around the globe - in real-time - is a necessary prerequisite for many applications, which include movement from one point to another. One particular field is the area of autonomous driving (AD) which opens up a lot of new and interesting applications. AD as a system innovation involves technological, social and infrastructural changes. Right now, several technical systems have been presented that operate on level two (partial automation) of the $\mathrm{SAE}^{1}$ scale. In order to reach the next level of automation, that also involves higher awareness of the automated vehicle, further technological innovations are needed. Here, robust and reliable GNSS is considered a key-technology. Moreover, the ongoing progress of urbanization causes a steady extension of satellite based navigation to urban canyon environments [2]. Within inner cities, users would like to navigate seamlessly without any obstacles in both outdoor and indoor [3].

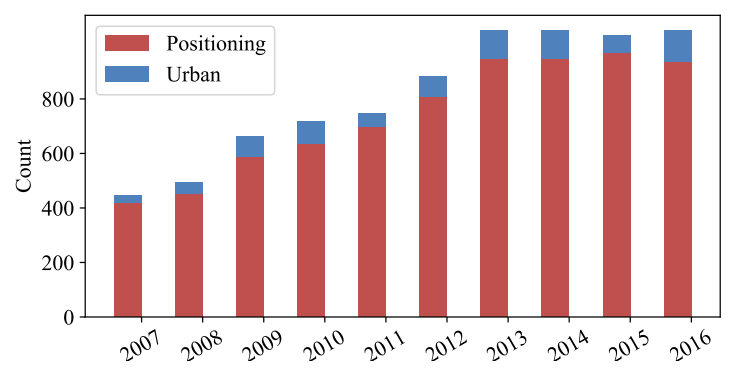

Figure 1: Number of relevant GNSS publications from 2007 to 2016 .

However, the dense urban area causes several errors to satellite navigation-based positioning and thus decreases the potential for various positioning applications [4]. Due to the fact that the urban environment provides perfect surfaces for reflections, non-line-of-sight (NLOS) situations are a source for the worst errors in cities. This effect is also known outside of cities such as airports [5] or in harsh natural environments such as mountains or forests with dense foliage.

\footnotetext{
*Julia Breßler, Technische Universität Chemnitz, 09107 Chemnitz, Germany \& julia.bressler@ wirtschaft.tu-chemnitz.de

${ }^{1} \mathrm{https} / / /$ www.sae.org/misc/pdfs/automated_driving.pdf
} 
Traditional approaches are not able to identify and eliminate several stochastic and deterministic errors in satellite based navigation under both, urban and indoor, conditions [6], [7]. In [8] various approaches to address the urban canyon problem by minimizing the negative influences to the navigation solution are described. Moreover, [4] presents four different methods for improving positioning performance and categorizes in

- usage of several common radio signals such as radio signals, Wi-Fi or new technologies such as Long Term Evolution (LTE) or 5G,

- integration of different sensors such as dead reckoning (DR), mainly low-cost inertial sensors and odometry data from vehicles [9],

- techniques for the detection or modeling of the surrounding environment of the localization object [10], [11], [12], [13].

However, no cost-efficient system which provides a reliable sub-meter-level positioning in urban areas based on one single method is known, today. Consequently, we expect that future applications will combine multiple solutions to increase the accuracy and reliability. That means the most sophisticated localization system requires the combination of the single advantages of heterogeneous techniques within one system.

According to [14], the main aspects of cost-efficient GNSS-based localization system are availability (e. g. by using more satellite systems [15]), accuracy [16] and reliability. Some approaches improve one particular aspect without evaluating the potential negative influence to the other criteria. We believe, than an innovative localization system should strive to increase all three aspects at once while keeping costs at a reasonable level. For this reason, navigation and real-time positioning in challenging environments still requires a lot of efforts in research [17] and industry.

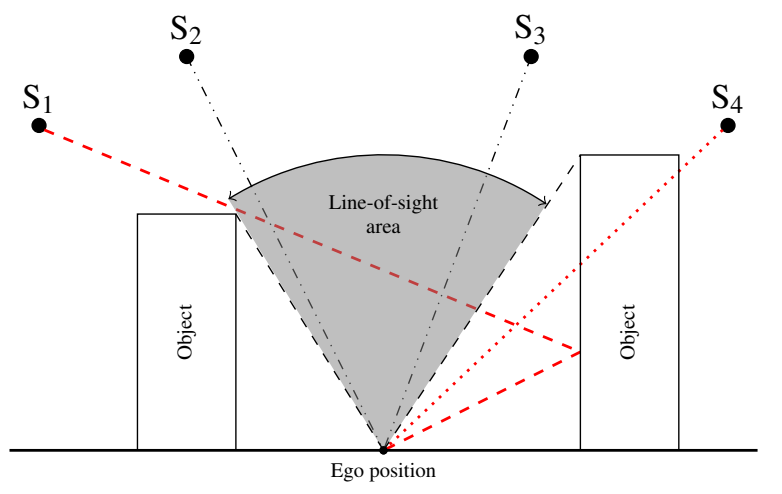

Figure 2: A typical situation in an urban area. The reception of each satellite signal depends on the position of the receiver and the satellite with respect to each other. Satellites $S_{2}$ and $S_{3}$ are in line-of-sight (LOS), satellite $S_{4}$ is blocked and satellite $S_{1}$ has non-line-of-sight (NLOS). This is a schematic representation of the electronic waves emitted by the satellites.
Based on [18], we focus on intelligent urban positioning (IUP) which includes new techniques for the detection of non-line-of-sight (NLOS) signal propagation. Thus, we will outline the current roadmap of scientists who deal with GNSS positioning under NLOS conditions. Within this survey, we intend to present a systematic overview of relevant data for NLOS handling. An explicitly in-depth assessment by benchmarking of the entire techniques is not aimed. However, we discuss topics such as measurement data generation. This survey is motivated, by the development of conference proceedings and journal publications of GNSS positioning as depicted in Figure 1, which has been stagnating since 2013. In fact, we present a subproblem of GNSS localization: the techniques for handling NLOS as well as the evaluation methods of the scientists. The paper is structure as as follows: in section 2 we first describe the NLOS phenomena by itself in order to prepare the introduction of an appropriate taxonomy. In section 3, we present a taxonomy of different NLOS handling techniques and evaluation methods. In the subsequent section an outlook to future trends and new approaches is given. The paper closes with a comprehensive conclusion in section 5 .

\section{Non-Line-of-sight}

When using GNSS localization there are various influencing factors, which might decrease the quality of a position estimate in urban scenarios. [8] claims two important challenges: Multipath and NLOS situations. Both phenomena appear in several application domains where electromagnetic wave propagation between a sender and a receiver is used. This includes the signal reflection from surrounding buildings, a poor satellite visibility or an unfavorable satellite constellation. For the sake of clarity, we deliberately distinguish between multipath and NLOS. If a satellite signal is received via multiple paths at the antenna, that is, via the direct path (also known as line-of-sight or LOS) and the NLOS path, it is characterized as multipath. If it is solely received via the reflection path it is simply characterized as NLOS. In inner cities with high buildings and narrow streets the receiver performance is usually affected by both, multipath and NLOS. Thus, both effects are the dominant sources for unwanted disturbances [19]. Figure 2 depicts a typical situation within an urban canyon. It is worthwhile to mention, that advanced antenna concepts (e.g. based on dual polarization [20], antenna arrays [21] or choke ring designs) are able to exclude the NLOS part from a multipath signal. However, this no longer works if only the NLOS signal is received. Moreover, these effects cannot be removed by differential techniques [7]. Thus, an efficient and explicit estimation/identification step is required.

In [14] the NLOS problem is divided into eight individual sectors. Based on the aforementioned work, we abstract three different levels: the technical design for the detection of NLOS; the objective of the approach itself; and the application domain (see Figure 3 .

- The technical design level consists of four different sub-levels-error analysis, hardware sensors, environmental modeling, fault detection/outlier classifi- 
cation. This level summarizes all technical implementations and designs.

- Objective includes the sub-levels handling and scope. It describes how the contained information within the NLOS signal is used. For example, if it is just ignored or exploited to a certain extend. Moreover, we separate between autonomous (stand-alone) and cooperative architectures.

- The level application domain comprises the main areas where GNSS-based localization techniques are used for the positioning process.

In the subsequent text, we use the combination of technical design and the level of objectives (especially handling) as categories for our survey. This includes the analysis of current NLOS methods under the aspects of availability, accuracy and reliability.

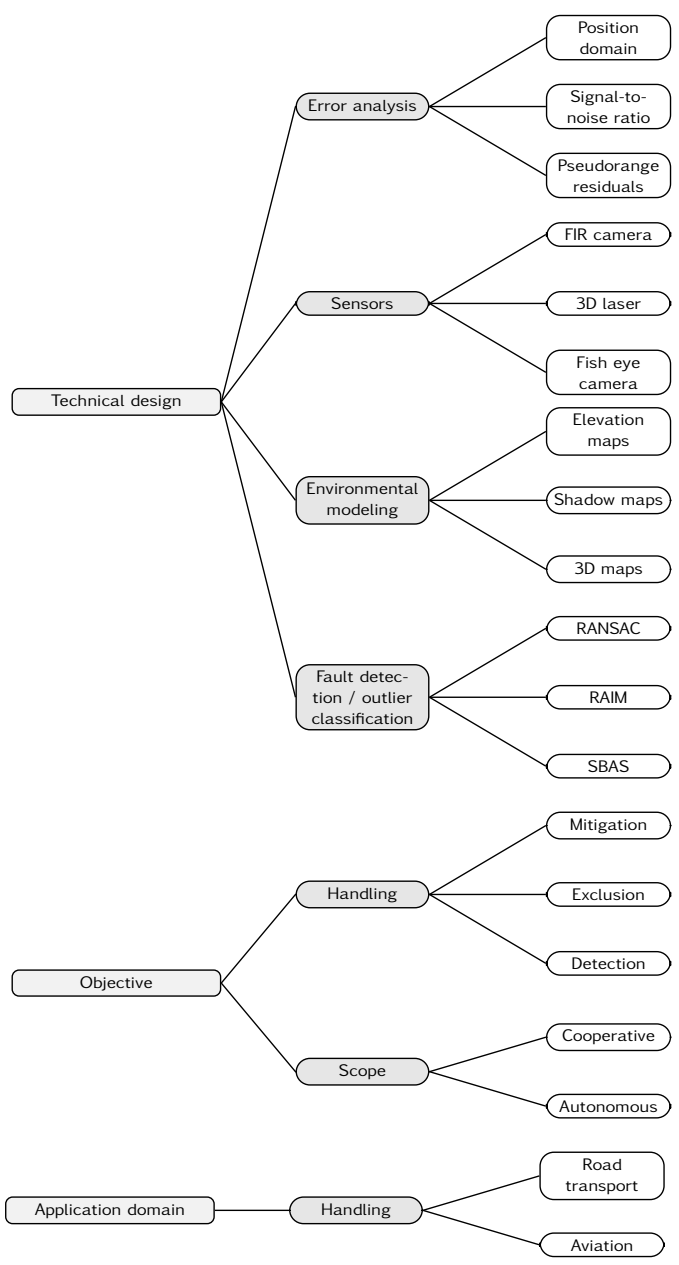

Figure 3: Overview of created taxonomy in reference to [14].

\section{Classification \& Taxonomy}

In this section we will create a taxonomy, in which the levels of handling, objectives and techniques are linked (see Figure 3). Based on these levels, we will assign different research activities. In addition, we provide two further categorizations based on an inductive approach. We summarize current research approaches, which lead us to the taxonomy stages. For the sake of clarity, we present a table which categorizes the evaluation methods found in literature. Finally, we present an overview that summarizes current approaches which address NLOS.

\subsection{Taxonomy of NLOS Handling Ap- proaches including Techniques}

After analyzing the literature, we came to the conclusion that there are four different high-level solutions to the NLOS problem. This includes: ignorance of the NLOS effect, mitigation of NLOS signals, identification of NLOS signals as well as avoiding NLOS. The latter one is equivalent to avoiding to go to cities or denied environments. Ignorance means, the disregard of multipath effects and NLOS signals at all. Although, this approach is not constructive in the sense of a modern localization system as highlighted in the introduction section, we included it for the sake of completeness. By mitigation we subsume approaches which basically assume that the effect of multipath can be minimized through the usage of improved features of hardware. In contrast, there is the field of identification. Here, we include approaches which aim to identify NLOS signals in order to use this information for further processing. In a second step, the identified NLOS signals can be eliminated or exploited.

It is worth mentioning that some approaches use different methods but generate similar results. For example, the sub-level receiver-based correlator design might mitigate the influence of NLOS without exact identification of the defect signal, while a similar work, which belongs to the sub-level modified receiver processing strategy, performs an identification followed by a subsequent elimination of that signal from the positioning process.

\section{Mitigation}

The work of [22] proposes two categories of mitigation techniques: antenna design and receiver-based correlator design. We augment this classification with two additional categories in our taxonomy, that is, weighting and using quality parameters. Due to the nature of these techniques, the algorithms are only able to decrease the influence of the NLOS error to the positioning solution without explicitly modeling which signals are received under NLOS or LOS conditions. Hence, they are for example not suitable for explicit modeling of pseudorange signal characteristics.

- Receiver-based correlator - different designs or implementations of the correlator at the HF-level are a key feature of work within this category and can be found in [23] and [24].

- Antenna design - based on a special design of the antenna, the NLOS part within a multipath signal can be extracted before the signal arrives the receiver. Representatives of this approach are: [8], [25], [26], [27] and [28]. Recurring on [8], six antenna designs can be distinguished, where the angle of arrival measurement (AOA)is rated the most performant technology. 
Although the AOA principle provides a high performance, it is worth mentioning that this antenna design is not widely adopted yet.

- Weighting - refers to the so-called elevation cut-off angle for different satellites. In general, low elevated satellites are more affected by NLOS than satellites with a high elevation. For this reason measurements from these satellites will be excluded before a position solution is computed. Implementations of that technique can be found in [29] and [30].

- Using quality parameters - the approaches in this category indirectly use different indicators such as signal-to-noise ratio (SNR), Doppler frequency, etc. to assess the probability of a measurement being a NLOS signal. Based on a user-defined threshold in this domain, the potential NLOS signals are either excluded or tagged in order to apply a weighting. Implementations of this can be found in [31], [32], [33], [34], [35], [36] and [37].

\section{Identification}

We split the top-level category identification (as proposed by [8] and [38]) into the sub-levels elimination and exploitation. For both sub-levels, the identification of NLOS signals is a necessary condition. Regarding the elimination of NLOS signals, the concept initially presented in [4] is extended with a further category named environmental modeling. Thus, the final four sub-levels of the category elimination are:

- Environmental modeling - describes the explicit modeling of potential external influences such as buildings or infrastructure of cities: [12], [39], [18], [40], [41], [42], [43], [44], [45], [46], [47], [48], [49], [50], [51]. We also propose to include approaches such as a sky-positioning camera within this category.

- Integration with inertial navigation systems (INSs) combines the benefits of two heterogeneous systems into one localization system: [14], [52], [53], [54], [55], [56], [57], [58].

- Modified receiver processing strategies - describes approaches were the receiver architectures is modified in order to achieve better signal processing aspects: [59], [60], [61], [62], [63], [64], [65], [66], [67], [68].

- Using multi-constellation GNSS - for increasing the overall amount of available satellite signals to choose from and thus improving the dilution of precision (DOP) metric: [13], [69], [70].

The level exploitation includes a large amount of approaches and consequently comprises several categories. The handling-technique is used to obtain further important information about accuracy, availability and reliability. Accordingly, the technique is appropriately utilized. This changes the view of NLOS: from a negative effect to an important information provider. Also, we have gathered techniques that allow to provide necessary information. In [43], the two categories signal-to-noise ratio and shadow matching are proposed. The category signal delay matching (SDM) was introduced in [4]. The other categories are taken from [14] and augmented with different collections of papers as shown in the following listing. It should be highlited, that recent research approaches usually propose a combination of the individual techniques. The categories of the sub-level exploitation are divided in:

- Signal-to-noise ratio - approaches sorted to this category aim to use the information about different SNR levels in several environments: [27], [47], [71], [72], [73], [74].

- Ray tracing - this technique uses several models (e.g. $3 \mathrm{D}$ city models) to predict an intersection of the LOS signal path with a building between a satellite and the receiver. In case of an intersection, the probability for NLOS is considered high: [4], [22], [71].

- SDM or signal delay matching - describes a technique to predict and observe parameters such as delta code phase delay. The observed parameter is then compared to a known model as shown in [4].

- Shadow matching - shadow matching is a pattern matching approach which utilizes a 3D city model in order to predict whether a satellite will be visible or not. Based on the decision whether a signal was directly received, the most likely position candidate from the 3D map can be selected: [11], [75], [76], [77], [78], [79] and [80].

- 3D building model - this level encompasses all approaches based on 3D building models which do not rely on ray tracing or shadow matching: [4], [22], [71], [81], [82], [83].

- Consistency checking - The rationale behind this approach is, that LOS measurements usually produce a more consistent navigation solution (e.g. in data fusion systems) than NLOS measurements [8]. Therefore, different combinations of satellite signals are used to compute individual position candidates which are afterwards compared to each other or another reference: [29], [84], [85], [86] and [87]. The technique of innovation filtering is also included in this category [8].

- Pseudorange residual - the least squares method aims to minimize the residuals produced by all considered measurements. The result is a more consistent (i.e. balanced) positioning solution as shown in [71].

- Carrier phase - this category is similar to the SNR level. However, this time the observed parameter is the carrier phase [88].

- Time delay and Doppler frequency - This category is similar to the SNR level. However, this time the observed parameters are the time delay and Doppler frequency: [89], [90] and [91]. 


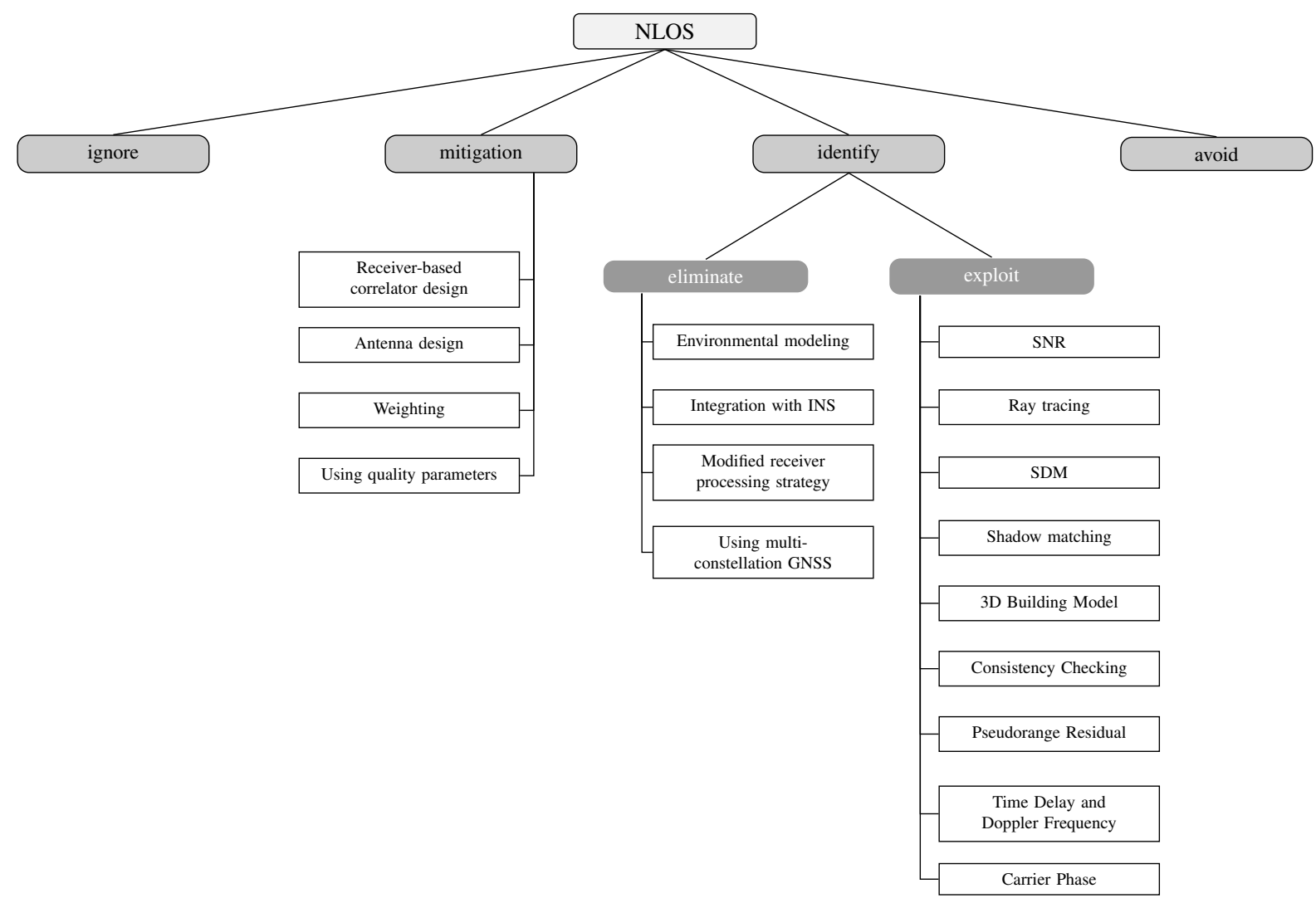

Figure 4: Classification of the NLOS problem into four different levels. Additionally, the level identify is divided into the two sub-levels eliminate and exploit. The different sub-levels are inspired by [4], [38], [8], [14] and extended by own categories.

Some approaches found in literature cannot be clearly assigned to one of the aforementioned categories. Moreover, papers which cover more than one technique are also missing at this stage. For the sake of completeness, we would like to add these papers to this survey. For example, techniques which utilize cooperative methods to exchange GNSS raw data in order to mitigate the influence of degraded satellite signals can be found in [92] and [93]. Other approaches try to detect jumps of the mean value within the measurement domain over time [94]. Moreover, various forms of receiver autonomous integrity monitoring (RAIM) have been investigated [95]. A further class of complex algorithms utilizes Dirichlet process mixtures (DPM) for multipath mitigation [96], [97].

\subsection{Evaluation Methods}

In the introduction, we mentioned the characterization of the scientific road map in the context of NLOS. Similarly, to the handling methods, we now want to present the analysis of the evaluation methods. At this point, we ask ourselves how the technical approaches for NLOS mitigation, identification and modeling are assessed. Therefore, we provide an overview of the used experimental setups. The focus is clearly on papers, which address NLOS or multipath handling. We proceed in an inductive manner. This means, we try to derive relevant categories from the reviewed papers. Within 53 papers we found static and dynamic measurement campaigns, which were destined for either vehicles (trucks, aircrafts, drones, cars, etc.) or persons. Moreover, these measurement campaigns can be categorized into simulation and real-world data collections. Table 1 illustrates our results and allows a classification. The row no information summarizes items that do not report the evaluation method. The classification is focused on real-world data collection for vehicles. We observed that real-world data collection campaigns for persons is constantly increasing since 2013. In contrast, simulations are underrepresented in connection with the exploration of identification and mitigation strategies of NLOS. We assume different obstacles in the process of transferring real world phenomena into a simulation model. The influences and dependencies between constantly changing environments during a dynamic maneuver are highly complex. Hence, the majority of the investigated papers use real-world data to cover most relevant side effects. During the characterization of the evaluation methods we also realized that a comparison of the results can hardly be produced. This is mainly due to a lack of a common dataset, which allows consistent benchmarking of the used methods and techniques.

\subsection{Recent solutions for positioning under NLOS conditions}

In this section, we will look at the different categories of our Survey for current research projects. Similarly, we test the statement by [8] that the combination of techniques is essential. The study of the reviewed work showed 7 research projects in the period from 2013 to 2015, which dealt with the NLOS problem. The methodology of the programs can 
be classified into the field of identification. Based on this current research, we analyzed solutions in form of technical design, scope and data collection. Due to this sampling we found:

- one approach with a cooperative scope [56]. This approach was evaluated by simulation.

- six approaches, which are specialized in environmental modeling with 3D ([4], [18], [22], [43], [47], [71]). Here, real-world data was collected. Thus, building boundary data, digital terrain models and building models were used.

Table 1: Types of Measurement Data

\begin{tabular}{|c|c|c|}
\hline $\begin{array}{l}\text { Real Data } \\
\text { collection }\end{array}$ & $\begin{array}{l}\text { Static } \\
\text { measurement }\end{array}$ & $\begin{array}{l}\text { Dynamic } \\
\text { measurement }\end{array}$ \\
\hline Via vehicle & $\begin{array}{lll}{[8],} & {[14],} & {[33],} \\
\lfloor[50], & {[80],} & {[98],} \\
\lfloor\overline{99]} & & \end{array}$ & $\begin{array}{lll}{[4],} & {[14],} & {[30]} \\
{[33]} & {[35],} & {[92],} \\
{[47],} & {[48],} & {[50]} \\
{[51]} & {[58],} & {[67]} \\
{[68],} & {[70],} & {[74]} \\
{[81]} & {[82],} & {[83]} \\
{[85]} & \end{array}$ \\
\hline $\begin{array}{l}\text { Via person } \\
\text { (No informa- } \\
\text { tion) }\end{array}$ & 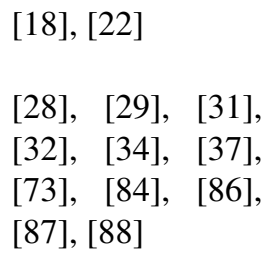 & $\begin{array}{l}\text { [43], [49], 66], } \\
{[71]}\end{array}$ \\
\hline Simulation & $\begin{array}{l}\text { Static } \\
\text { measurement }\end{array}$ & $\begin{array}{l}\text { Dynamic } \\
\text { measurement }\end{array}$ \\
\hline Via vehicle & [90] & $\begin{array}{l}{[14],[27], \quad[56],} \\
{[68],[91],[94]}\end{array}$ \\
\hline $\begin{array}{l}\text { Via person } \\
\text { (No informa- } \\
\text { tion) }\end{array}$ & \multicolumn{2}{|c|}{ [25], [41], [89], [95] } \\
\hline
\end{tabular}

Furthermore, this analysis reveals that these projects used a combination of technical designs in order to identify the NLOS signal. For example, [18] used building models, consistency checking, signal geometry and signal strength information within one algorithm. In addition, we were able to determine which criteria were used to evaluate the research projects. In all studies, accuracy was the main objective, while some studies focused on the availability aspect as well. Only four studies addressed the performance indicator reliability.

Furthermore, an assumption from our introduction was confirmed. Thus, the costs represent a significant influencing factor on the techniques, which were used. Four out of the seven research programs address the low-cost aspect: [4], [18], [43] and [47]. For this reason, we conducted further meta-analysis on the criteria for NLOS handling techniques by investigating the following sources: conference proceedings as well as journal publication from the years 2013 to 2016. It became clear that accuracy was given to
$59 \%$, availability to $27 \%$ and reliability to $14 \%$ as observation and influencing factors for the assessment of NLOS approaches. Figure 5 illustrates this evolution. In addition, we observed an increase in low-cost conditions for NLOS techniques since 2014. Basically, these criteria can be considered evaluation standards for a common benchmarking of NLOS handling techniques.

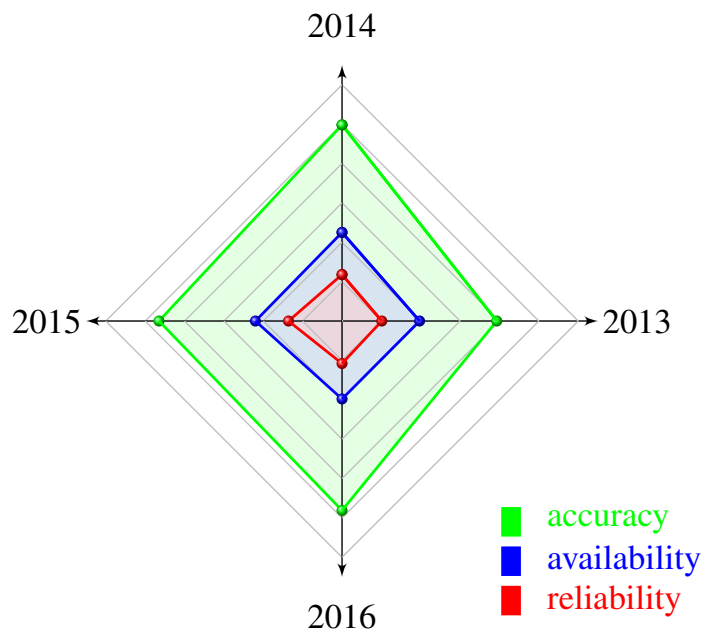

Figure 5: Evolution of evaluation criteria from 2013 to 2016.

\section{Current Development}

Despite the steadily growing field of satellite navigation for pedestrians, in combination with other techniques a seamless navigation independent from the environments, the majority of usage is for various vehicles on roads. This fact is shown in the rows for vehicle of Table 1 . On the other hand, there are some gaps of research and experience with satellite-based navigation for pedestrians. Another fact is the ongoing development of autonomous vehicles in the near future. A reliable and accurate navigation system is one of the enabling-technologies for improved mobility. For this purpose the vehicle have to trust the navigation solution, independently of harsh environments or good visibility of satellites. In theory, there are several techniques to fulfill this task. However, we expect that the combination of multiple approaches will result in a safe navigation system that can be used in case of outage or partial degradation of a sub-system.

Although, this survey paper focuses on an analysis of the state-of-the-art of GNSS positioning under NLOS conditions, we would like to mention that there are a couple of national and European initiatives which aim to improve satellite navigation in challenging areas. For example, the goal of the German research project smartLof ${ }^{2}$ is to develop a robust positioning algorithm based on the factor graphs framework as used in the robotics community. The main motivation is to enable low-cost receivers to be used in urban areas by deliberately modeling the LOS and NLOS statistics inside of the navigation algorithm. By that ap-

${ }^{2}$ http://www.smartLoc.eu 
proach, an appropriate integrity as well as an improve accuracy is expected. The algorithm is designed to explicitly handle temporal mulit-modalities of the GPS pseudoranges as caused under NLOS conditions. Similarly, the European research project InDrive ${ }^{3}$ proposes to use a software GNSS receiver in combination with a Bayesian data fusion framework in order to estimate and identify NLOS signals already at the correlator level. In contrast to a standard GNSS receiver which typically solely emits one PVT solution per epoch, the InDrive concept allows to provide multiple solutions in case of temporal ambiguities. Due to the probabilistic interface on the output side, later stages (e.g. inside of a complex system architecture as in autonomous vehicles) can be used to finally select the most appropriate solution candidate based on further information (e.g. from digital maps or other exteroceptive sensors).

\section{Conclusion}

In this paper we addressed a specific segment or phenomenon of robust localization: NLOS. With our survey approach, we intend to present a systematic search for specific data relevant to the NLOS handling. Starting from the work presented in [14] and an analysis of several papers we have developed a taxonomy of handling NLOS in GNSS positioning. Thereby we integrated the fields of handling, the technology used and the intended objective. Afterwards, we identified types of evaluation methods and analyzed which are primarily used. Finally, we looked at current research programs and their technical design, data collection, handling, scope and application domain.

The output of Table 1 and the classification in Figure 4 give a good impression of the usage of satellite-based navigation. The majority of the reviewed papers are related to outdoor environments for any kind of vehicles. Many of them used real-world data to compare their solutions. As the used reference data (ground truth) is not the same within the data sets, an absolute comparison regarding the algorithmic performance is difficult. Hence, a quantitative comparison of the various solutions should be addressed in future work. Then, we applied the taxonomy of handling and evaluation methods to current research projects. It became clear that cooperative handling approaches as well as real-data measurements belong to the current standard. In addition, we were able to characterize the evaluation criteria of the researchers for their chosen technique and to outline an essential condition for the technique. Thus, we could determine the current researcher roadmap for dealing with NLOS. The roadmap is defined by the choice of several cooperating NLOS handling techniques in coordination with the respective research objective (mitigation, exploitation or elimination). Then, the evaluation of this technique is carried out under the conditions of low-cost, due to the availability, accuracy and reliability of the GNSS localization system. Real data are usually used for this purpose. In the future, we would like to use a common dataset to benchmark the used methods and techniques. These steps are needed to create technological innovations for our industrial and globally connected world - especially for sys- tem innovations in the area of ITS.

Conflict of Interest The authors declare no conflict of interest.

Acknowledgment We want to thank Pierre Reisdorf for his contributions to the initial ITSC conference paper.

\section{References}

[1] J. Breßler, P. Reisdorf, M. Obst, and G. Wanielik, "Gnss positioning in non-line-of-sight context a survey," in Intelligent Transportation Systems (ITSC), 2016 IEEE 19th International Conference on. IEEE, 2016, pp. 1147-1154.

[2] R. Ercek, P. D. Doncker, and F. Grenez, "Study of Pseudo-Range Error Due to Non-Line-of-Sight-Multipath in Urban Canyons," in Proceedings of the 18th International Technical Meeting of the Satellite Division of The Institute of Navigation (ION GNSS 2005), 2005, pp. 1083 - 1094.

[3] J. R. A. Watson, "High-Sensitivity GPS L1 Signal Analysis for Indoor Channel Modeling," Master's thesis, The University of Calgary, 2005.

[4] R. Kumar and M. G. Petovello, "A Novel GNSS Positioning Technique for Improved Accuracy in Urban Canyon Scenarios Using 3D City Model," in Proceedings of the 27th International Technical Meeting of The Satellite Division of the Institute of Navigation (ION GNSS+ 2014), 2014, pp. 2139 - 2148.

[5] A. Guilloton, A. C. Escher, and D. Koenig, "Multipath study on the airport surface," in Position Location and Navigation Symposium (PLANS), 2012 IEEE/ION, April 2012, pp. 355 - 365.

[6] P. Misra and P. Enge, Global Positioning System: Signals, Measurements, and Performance. Ganga-Jamuna Press, 2011.

[7] P. W. Ward, J. W. Betz, and C. J. Hegarty, Understanding GPS: Principles and Applications, Second Edition. Artech House, 2006, ch. Chapter 6: Interference, Multipath, and Scintillation.

[8] P. D. Groves, Z. Jiang, M. Rudi, and P. Strode, "A Portfolio Approach to NLOS and Multipath Mitigation in Dense Urban Areas," in Proceedings of the 26th International Technical Meeting of The Satellite Division of the Institute of Navigation (ION GNSS 2013), 2013, pp. 3231 - 3247.

[9] P. Groves, Principles of GNSS, Inertial, and Multisensor Integrated Navigation Systems, Second Edition, 2nd ed., ser. GNSS/GPS. Artech House, 2013.

[10] P. Furgale and et al., "Toward automated driving in cities using closeto-market sensors: An overview of the V-Charge Project," in Intelligent Vehicles Symposium (IV), 2013 IEEE, June 2013, pp. $809-$ 816.

[11] L. Wang, P. Groves, and M. Ziebart, "Urban Positioning on a Smartphone: Real-time Shadow Matching Using GNSS and 3D City Models," in Proceedings of the 26th International Technical Meeting of The Satellite Division of the Institute of Navigation (ION GNSS+ 2013), 2013, pp. 1606 - 1619.

[12] A. Bourdeau, M. Sahmoudi, and J.-Y. Tourneret, "Tight Integration of GNSS and a 3D City Model for Robust Positioning in Urban Canyons," in Proceedings of the 25th International Technical Meeting of The Satellite Division of the Institute of Navigation (ION GNSS 2012), 2012, pp. 1263 - 1269.

[13] P. D. Groves, "Shadow Matching: A New GNSS Positioning Technique for Urban Canyons," Journal of Navigation, vol. 64, no. 03, pp. 417-430, 2011.

[14] M. Obst, "Bayesian Approach for Reliable GNSS-based Vehicle Localization in Urban Areas," Ph.D. dissertation, Technische Universität Chemnitz, 2015.

[15] C. J. Hegarty, "GNSS Signals-An Overview," in 2012 IEEE International Frequency Control Symposium Proceedings, May 2012, pp. $1-7$.

${ }^{3}$ http://www.indrive-project.eu/ 
[16] F. van Diggelen, "GNSS Accuracy-Lies, Damn Lies and Statistics," GPS World, vol. 18, no. 1, Jan. 2007. [Online]. Available: http://www.frankvandiggelen.com/wp-content/uploads/2009/ 03/2007-gps-world-accuracy-article-0107-van-diggelen-1.pdf

[17] P. D. Groves, L. Wang, D. Walter, H. Martin, K. Voutsis, and Z. Jiang, "The Four Key Challenges of Advanced Multisensor Navigation and Positioning," in 2014 IEEE/ION Position, Location and Navigation Symposium - PLANS 2014, May 2014, pp. 773 - 792.

[18] M. Adjrad and P. D. Groves, "Enhancing Conventional GNSS Positioning with 3D Mapping without Accurate Prior Knowledge," in Proceedings of the 28th International Technical Meeting of The Satellite Division of the Institute of Navigation (ION GNSS+ 2015) September 14 - 18, 2015 Tampa Convention Center Tampa, Florida, 2015.

[19] M. S. Braasch, "Performance comparison of multipath mitigating receiver architectures," in Aerospace Conference, 2001, IEEE Proceedings., vol. 3, 2001, pp. 3/1309 - 3/1315 vol.3.

[20] P. D. Groves, Z. Jiang, B. Skelton, P. A. Cross, L. Lau, Y. Adane, and I. Kale, "Novel Multipath Mitigation Methods Using a DualPolarization Antenna," 2010.

[21] J. Ray, M. Cannon, and P. Fenton, "GPS Code and Carrier Multipath Mitigation Using a Multiantenna System," IEEE Transactions on Aerospace and Electronic Systems, vol. 37, no. 1, pp. 183-195, 2001 .

[22] L.-T. Hsu, Y. Gu, and S. Kamijo, "3D building model-based pedestrian positioning method using GPS/GLONASS/QZSS and its reliability calculation," GPS Solutions, pp. 1-16, 2015.

[23] L. Garin, F. van Diggelen, and J.-M. Rousseau, "Strobe \& Edge Correlator Multipath Mitigation for Code," in Proceedings of the 9th International Technical Meeting of the Satellite Division of The Institute of Navigation (ION GPS 1996), 1996, pp. 657 - 664.

[24] A. J. V. Dierendonck, P. Fenton, and T. Ford, "Theory and Performance of Narrow Correlator Spacing in a GPS Receiver," NAVIGATION, Journal of the Institute of Navigation, vol. 39, no. 3, pp. 115 $-124,1992$

[25] G. A. McGraw, R. S. Young, K. Reichenauer, J. Stevens, and F. Ventrone, "GPS Multipath Mitigation Assessment of Digital Beam Forming Antenna Technology in a JPALS Dual Frequency Smoothing Architecture," in Proceedings of the 2004 National Technical Meeting of The Institute of Navigation, 2004, pp. 561 - 572.

[26] M. S. Braasch, "Multipath effects," in Global Positioning System: Theory and Applications, 1996.

[27] O. Osechas, K. J. Kim, K. Parsons, and Z. Sahinoglu, "Detecting Multipath Errors in Terrestrial GNSS Applications," in Proceedings of the 2015 International Technical Meeting of The Institute of Navigation, 2015.

[28] M. H. Keshvadi, A. Broumandan, and G. Lachapelle, "Analysis of GNSS Beamforming and Angle of Arrival Estimation in Multipath Environments," Proceedings of the Institute of Navigation 2011 International Technical Meeting (ION ITM), San Diego, CA, USA, pp. $24-26,2011$

[29] P. D. Groves and Z. Jiang, "Height Aiding, $\mathrm{C} / \mathrm{N}_{0}$ Weighting and Consistency Checking for GNSS NLOS and Multipath Mitigation in Urban Areas," Journal of Navigation, vol. 66, no. 05, pp. $653-669$, 2013.

[30] O. Le Marchand, P. Bonnifait, J. Bañez-Guzmán, F. Peyret, and D. Betaille, "Performance Evaluation of Fault Detection Algorithms as Applied to Automotive Localisation," in European Navigation Conference - GNSS 2008, Toulouse, France, Apr. 2008.

[31] C. Rost and L. Wanninger, "Carrier Phase Multipath Mitigation Based on GNSS Signal Quality Measurements," Journal of Applied Geodesy, vol. 3, no. 2, pp. 81 - 87, 2009.

[32] A. Bilich, K. M. Larson, and P. Axelrad, "Modeling GPS phase multipath with SNR: Case study from the Salar de Uyuni, Boliva," Journal of Geophysical Research (Solid Earth), vol. 113, p. B04401, Apr. 2008

[33] C. J. Comp and P. Axelrad, "Adaptive SNR-based Carrier Phase Multipath Mitigation Technique," IEEE Transactions on Aerospace and Electronic Systems, vol. 34, no. 1, pp. 264 - 276, Jan 1998.
[34] A. Hirrle, H.-J. Jentschel, and O. Michler, "Estimation of Multipath Parameters using Hilbert Huang Transform," in Proceedings of the 25th International Technical Meeting of The Satellite Division of the Institute of Navigation (ION GNSS 2012), September 2012, pp. 67 78.

[35] M. Kim, K. Kim, J. Lee, and S. Pullen, "High Integrity GNSS Nav igation and Safe Separation Distance to Support Local-Area UAV Networks," in Proceedings of the 27th International Technical Meeting of The Satellite Division of the Institute of Navigation (ION GNSS+ 2014), 2014, pp. $869-878$.

[36] O. Le Marchand, P. Bonnifait, J. Ibanez-Guzmán, D. Betaille, and F. Peyret, "Characterization of GPS multipath for passenger vehicles across urban environments," ATTI dell'Istituto Italiano di Navigazione, no. 189, pp. 77 - 88, 2009.

[37] L. Li, J. Zhong, and M. Zhao, "Doppler-Aided GNSS Position Estimation With Weighted Least Squares," IEEE Transactions on Vehicular Technology, vol. 60, no. 8, pp. 3615 - 3624, Oct 2011.

[38] Z. Jiang and P. D. Groves, "NLOS GPS signal detection using a dualpolarisation antenna," GPS solutions, vol. 18, no. 1, pp. $15-26$, 2014

[39] L. Wang, P. D. Groves, and M. K. Ziebart, "Multi-Constellation GNSS Performance Evaluation for Urban Canyons using Large Virtual Reality City Models,' Journal of Navigation, vol. 65, no. 03, pp. 459 - 476, 2012

[40] Y. Suh and R. Shibasaki, "Evaluation of Satellite-Based Navigation Services in Complex Urban Environments Using a ThreeDimensional GIS," Ieice Transactions, vol. 90-B, pp. 1816 - 1825, 2007.

[41] J. Bradbury, M. Ziebart, and P. Cross, "Code Multipath Modelling in the Urban Environment Using Large Virtual Reality City Models: Determining the Local Environment," The Journal of Navigation, vol. 60, pp. 95 - 105, 2007.

[42] T. Suzuki and N. Kubo, "Correcting GNSS Multipath Errors Using a 3D Surface Model and Particle Filter," in Proceedings of the 26th International Technical Meeting of The Satellite Division of the Institute of Navigation (ION GNSS 2013), 2013, pp. 1583 - 1595.

[43] A. T. Irish, D. Iland, J. T. Isaacs, J. a. P. Hespanha, E. M. Belding, and U. Madhow, "Using Crowdsourced Satellite SNR Measurements for 3D Mapping and Real-time GNSS Positioning Improvement," in Proceedings of the 6th Annual Workshop on Wireless of the Students, by the Students, for the Students, ser. S3 '14. New York, NY, USA: ACM, 2014, pp. 5 - 8.

[44] M. Obst, S. Bauer, and G. Wanielik, "Urban Multipath Detection and Mitigation with Dynamic 3D Maps for Reliable Land Vehicle Localization," in Position Location and Navigation Symposium (PLANS), 2012 IEEE/ION, April 2012, pp. 685 - 691

[45] P. D. Groves, Z. Jiang, L. Wang, and M. K. Ziebart, "Intelligent Urban Positioning Using Multi-constellation GNSS with 3D Mapping and NLOS Signal Detection," in ION GNSS 2012, 2012, pp. $458-$ 472

[46] B. Ben-Moshe, E. Elkin, H. Levi, and A. Weissman, "Improving Accuracy of GNSS Devices in Urban Canyons," in CCCG, 2011.

[47] S. Peyraud, D. Bétaille, S. Renault, M. Ortiz, F. Mougel, D. Meizel, and F. Peyret, "About Non-Line-Of-Sight Satellite Detection and Exclusion in a 3D Map-Aided Localization Algorithm," Sensors, vol. 13 , no. 1, p. 829,2013

[48] F. Peyret, D. , Bétaille, and F. Mougel, "Non-Line-Of-Sight GNSS Signal Detection Using an On-Board 3D Model of Buildings," in ITS Telecommunications (ITST), 2011 11th International Conference on. IEEE, 2011, pp. 280 - 286.

[49] L.-T. Hsu, Y. Gu, and S. Kamijo, "NLOS Correction/Exclusion for GNSS Measurement Using RAIM and City Building Models," Sensors, vol. 15 , no. 7 , p. $17329,2015$.

[50] J. i. Meguro, T. Murata, J. i. Takiguchi, Y. Amano, and T. Hashizume, "GPS Multipath Mitigation for Urban Area Using Omnidirectional Infrared Camera," IEEE Transactions on Intelligent Transportation Systems, vol. 10, no. 1, pp. 22 - 30, March 2009.

[51] F. Peyret, D. Betaille, M. Ortiz, S. Miquel, and L. Fontenay, "How to Improve GNSS Positioning Quality of Service for Demanding ITS in City Environments by Using 3D Digital Maps," in 19th ITS World Congress, 2012. 
[52] M. Petovello, C. O'Driscoll, and G. Lachapelle, "Weak Signal Carrier Tracking using Extended Coherent Integration with an UltraTight GNSS/IMU Receiver," Proc. ENC-GNSS 2008, 2008.

[53] M. Petovello and G. Lachapelle, "Comparison of Vector-Based Software Receiver Implementations with Application to UltraTight GPS/INS Integration," Proceedings of Institute of Navigation GPS/GNSS Conference, Fort Worth, TX. Institute of Navigation, 2006.

[54] A. Soloviev and F. V. Graas, "Use of Deeply Integrated GPS/INS Architecture and Laser Scanners for the Identification of Multipath Reflections in Urban Environments," IEEE Journal of Selected Topics in Signal Processing, vol. 3, no. 5, pp. 786 - 797, Oct 2009.

[55] A. Soloviev, C. Toth, and D. Grejner-Brzezinska, "Performance of Deeply Integrated GPS/INS in Dense Forestry Areas," in Proceedings of the 24th International Technical Meeting of The Satellite Division of the Institute of Navigation (ION GNSS 2011), 2011, pp. $2427-2435$.

[56] J. W. Cheong, E. Glennon, A. G. Dempster, D. Serant, and T. Calmettes, "Modelling and Mitigating Multipath and NLOS for Cooperative Positioning in Urban Canyons," 2015.

[57] P. D. Groves, C. J. Mather, and A. A. Macaulay, "Demonstration of Non-coherent Deep INS/GPS Integration for Optimised Signal-toNoise Performance," 2007.

[58] E. López Casariego, E. Pérez, G. Falco, M. Campo-Cossío Gutiérrez, F. Zacchello, and S. Bories, "Low-cost Real-time Tightly-coupled GNSS/INS Navigation System Based on Carrier Phase Double Differences for UAV Applications," in Proceedings of the 27th International Technical Meeting of The Satellite Division of the Institute of Navigation (ION GNSS+ 2014), Tampa, Florida, USA, 09/2014 2014, pp. $841-857$.

[59] P. Xie and M. G. Petovello, "Improving High Sensitivity Receiver Performance in Multipath Environment for Vehicular Applications," in Proceedings of the 25th International Technical Meeting of the Satellite Division of the Institute of Navigation (ION GNSS 2012), Nashville, TN, USA, vol. 1721, 2012, p. 448458.

[60] P. Xie, M. G. Petovello, and C. Basnayake, "Multipath Signal Assessment in the High Sensitivity Receivers for Vehicular Applications," in Proceedings of the ION GNSS, 2011.

[61] L. R. Weill, "A High Performance Code and Carrier Tracking Architecture for Ground-Based Mobile GNSS Receivers," in Proceedings of the ION GNSS Conference, 2010.

[62] F. van Graas, A. Soloviev, M. U. de Haag, and S. Gunawardena, "Closed-Loop Sequential Signal Processing and Open-Loop Batch Processing Approaches for GNSS Receiver Design," IEEE Journal of Selected Topics in Signal Processing, vol. 3, no. 4, pp. 571 - 586, Aug 2009.

[63] M. Petovello, C. O'Driscoll, and G. Lachapelle, "Carrier Phase Tracking of Weak Signals Using Different Receiver Architectures," in Proceedings of the ION NTM Conference, San Diego, CA, USA, vol. 2830, 2008, p. 781791.

[64] M. Uijt de Haag, "An Investigation into the Application of Block Processing Techniques for the Global Positioning System," Ph.D. dissertation, Ohio University, 1999.

[65] D. Flament, D. Brocard, W. Ochieng, and C. Milner, "RAIM in dual frequency / multi constellation APV/LPV operations in aeronautics," in 2010 5th ESA Workshop on Satellite Navigation Technologies and European Workshop on GNSS Signals and Signal Processing (NAVITEC), Dec 2010, pp. 1 - 7.

[66] L.-T. Hsu, P. D. Groves, and S.-S. Jan, "Assessment of the Multipath Mitigation Effect of Vector Tracking in an Urban Environment," 2013.

[67] M. Kaddour, N. A. Tmazirte, M. E. E. Najjar, Z. Naja, and N. Moubayed, "Fault detection and exclusion of cycle slips for carrier-phase in GNSS positioning," in Information Fusion (Fusion), 2015 18th International Conference on, July 2015, pp. 393 - 398.

[68] J. Marais, E. Duflos, N. Viandier, D. F. Nahimana, and A. Rabaoui, "Advanced signal processing techniques for multipath mitigation in land transportation environment," in Intelligent Transportation Systems (ITSC), 2010 13th International IEEE Conference on, Sept 2010, pp. $1480-1485$.
[69] C. O'Driscoll, G. Lachapelle, and M. Tamazin, "Investigation of the Benefits of Combined GPS/Glonass Receivers in Urban Environments," in Proceeding on RIN NAV10 Conference on Position, Location, Timing: Everyone, Everything, Everywhere, 2010.

[70] S. Feng, S. Wang, and W. Ochieng, "A Core Constellation Based Multiple-GNSS Positioning and Integrity Monitoring Algorithm," in Proceedings of the 27th International Technical Meeting of The Satellite Division of the Institute of Navigation (ION GNSS+ 2014), 2014, pp. $307-314$.

[71] S. Miura and S. Kamijo, "GPS Error Correction by Multipath Adaptation," International Journal of Intelligent Transportation Systems Research, vol. 13, no. 1, pp. 1-8, 2014.

[72] M. Obst and G. Wanielik, "Probabilistic Non-Line-of-Sight Detection in Reliable Urban GNSS Vehicle Localization Based on an Empirical Sensor Model," in Intelligent Vehicles Symposium (IV), 2013 IEEE, June 2013, pp. 363 - 368.

[73] A. L. Bilich, "Improving the precision and accuracy of geodetic GPS: applications to multipath and seismology," Ph.D. dissertation, University of Colorado, 2006.

[74] S. Bauer, R. Streiter, and G. Wanielik, "Non-line-of-sight mitigation for reliable urban gnss vehicle localization using a particle filter," in Information Fusion (Fusion), 2015 18th International Conference on, July 2015, pp. 1664-1671.

[75] L. Wang, P. D. Groves, and M. K. Ziebart, "GNSS Shadow Matching: Improving Urban Positioning Accuracy Using a 3D City Model with Optimized Visibility Prediction Scoring," in Proceedings of the 25th International Technical Meeting of The Satellite Division of the Institute of Navigation (ION GNSS 2012), 2012, pp. 423 - 437.

[76] R. Yozevitch, B. Ben-Moshe, and A. Dvir, "GNSS Accuracy Improvement Using Rapid Shadow Transitions," IEEE Transactions on Intelligent Transportation Systems, vol. 15, no. 3, pp. 1113 - 1122, June 2014.

[77] J. T. Isaacs, A. T. Irish, F. Quitin, U. Madhow, and J. P. Hespanha, "Bayesian Localization and Mapping Using GNSS SNR Measurements," in 2014 IEEE/ION Position, Location and Navigation Symposium - PLANS 2014, May 2014, pp. 445 - 451.

[78] L. Wang, P. D. Groves, and M. K. Ziebart, "Smartphone Shadow Matching for Better Cross-street GNSS Positioning in Urban Environments," Journal of Navigation, vol. 68, pp. 411 - 433, 52015.

[79] R. Yozevitch and B. B. Moshe, "A Robust Shadow Matching Algorithm for GNSS Positioning," in NAVIGATION, Journal of the Institute of Navigation, vol. 62, no. 2, 2015, pp. 95 - 109.

[80] P. D. Groves, Z. Jiang, L. Wang, and M. Ziebart, "Intelligent Urban Positioning, Shadow Matching and Non-Line-of-Sight signal detection," in Satellite Navigation Technologies and European Workshop on GNSS Signals and Signal Processing, (NAVITEC), 2012 6th ESA Workshop on, Dec 2012, pp. $1-8$.

[81] D. Betaille, F. Peyret, M. Ortiz, S. Miquel, and L. Fontenay, "A New Modeling Based on Urban Trenches to Improve GNSS Positioning Quality of Service in Cities," Intelligent Transportation Systems Magazine, IEEE, vol. 5, no. 3, pp. 59 - 70, 2013.

[82] V. Drevelle and P. Bonnifait, "Global positioning in urban areas with 3-D maps," in Intelligent Vehicles Symposium (IV), 2011 IEEE, June 2011, pp. $764-769$.

[83] V. Drevelle and P. Bonnifait, "iGPS: Global Positioning in Urban Canyons with Road Surface Maps," IEEE Intelligent Transportation Systems Magazine, vol. 4, no. 3, pp. 6 - 18, 2012.

[84] Z. Jiang, P. D. Groves, W. Y. Ochieng, S. Feng, C. D. Milner, and P. G. Mattos, "Multi-Constellation GNSS Multipath Mitigation Using Consistency Checking," Proceedings of the 24th International Technical Meeting of The Satellite Division of the Institute of Navigation (ION GNSS 2011), pp. 3889 - 3902, 2011.

[85] N. Alam, A. T. Balaei, and A. G. Dempster, "Relative Positioning Enhancement in VANETs: A Tight Integration Approach," IEEE Transactions on Intelligent Transportation Systems, vol. 14, no. 1, pp. 47 - 55, March 2013.

[86] H. Isshiki, "A New Method for Detection, Identification and Mitigation of Outliers in Receiver Autonomous Integrity Monitoring (RAIM)," in Proceedings of the 26th International Technical Meeting of The Satellite Division of the Institute of Navigation (ION GNSS 2013), 2013, pp. $151-158$. 
[87] Z. Jiang and P. D. Groves, "GNSS NLOS and Multipath Error Mitigation using Advanced Multi-Constellation Consistency Checking with Height Aiding," 2012.

[88] R. Moradi, W. Schuster, S. Feng, A. Jokinen, and W. Ochieng, "The carrier-multipath observable: a new carrier-phase multipath mitigation technique," GPS Solutions, vol. 19, no. 1, pp. 73 - 82, 2014.

[89] J. Li, R. Wu, D. Lu, and W. Wang, "GPS Multipath Mitigation Algorithm Based on Signal Correlation Character," International Journal of Digital Content Technology and its Applications(JDCTA), vol. 7, no. 3, pp. 837 - 846, February 2013.

[90] J. Li, Y. Li, and Y. Zhou, The Proceedings of the Second International Conference on Communications, Signal Processing, and Systems. Cham: Springer International Publishing, 2014, ch. GPS Multipath Mitigation Algorithm Using C/A Code Correlation Character, pp. $1047-1058$.

[91] P. Closas, C. Fernandez-Prades, and J. A. Fernandez-Rubio, "A Bayesian Approach to Multipath Mitigation in GNSS Receivers," IEEE Journal of Selected Topics in Signal Processing, vol. 3, no. 4, pp. 695 - 706, Aug 2009.

[92] N. Mattern, M. Obst, R. Schubert, and G. Wanielik, "Simulative Analysis of Accuracy Demands of Co-Operative Localization in the COVEL Project," in Intelligent Vehicles Symposium (IV), 2011 IEEE, June 2011, pp. 516 - 521.

[93] F. de Ponte Müller, E. M. Diaz, B. Kloiber, and T. Strang, "Bayesian cooperative relative vehicle positioning using pseudorange differences," in 2014 IEEE/ION Position, Location and Navigation Symposium - PLANS 2014, May 2014, pp. 434 - 444.
[94] M. Spangenberg, J. Y. Tourneret, V. Calmettes, and G. Duchateau, "Detection of Variance Changes and mean Value Jumps in Measurement Noise for Multipath Mitigation in Urban Navigation," in 2008 42 d Asilomar Conference on Signals, Systems and Computers, Oct 2008, pp. 1193 - 1197.

[95] J. Wang and P. B. Ober, "On the Availability of Fault Detection and Exclusion in GNSS Receiver Autonomous Integrity Monitoring," Journal of Navigation, vol. 62, no. 02, pp. 251 - 261, 2009.

[96] A. Rabaoui, N. Viandier, J. Marais, and E. Duflos, "Using Dirichlet Process Mixtures for the Modelling of GNSS Pseudorange Errors in Urban Canyon," in Proceedings of the 22nd International Technical Meeting of The Satellite Division of the Institute of Navigation (ION GNSS 2009), 2009, pp. $2391-2399$.

[97] A. Rabaoui, N. Viandier, J. Marais and E. Duflos, "On the use of Dirichlet process mixtures for the modelling of pseudorange errors in multi-constellation based localisation," in Intelligent Transport Systems Telecommunications,(ITST), 2009 9th International Conference on, Oct 2009, pp. $465-470$.

[98] P. Axelrad, C. J. Comp, and P. F. Macdoran, "SNR-Based Multipath Error Correction for GPS Differential Phase," IEEE Transactions on Aerospace and Electronic Systems, vol. 32, no. 2, pp. 650 - 660, April 1996.

[99] L. Serrano, "Carrier-Phase Multipath Mitigation In RTK-Based GNSS Dual-Antenna Systems," Ph.D. dissertation, Ph.D. dissertation, Department of Geodesy and Geomatics Engineering, Technical Report No. 287, University of New Brunswick, Fredericton, New Brunswick, Canada, 227 pp., 2013. 